\title{
L'usage de statines chez des patients au faible risque de maladies cardio-vasculaires présente un rapport coût-utilité défavorable
}

\author{
Swiss Medical Board a publié de nouvelles recommandations concernant l'usage de \\ statines en prévention primaire des maladies cardio-vasculaires. Dans son rapport, \\ le Conseil d'experts arrive à la conclusion qu'avant de prescrire des statines, il est \\ indispensable d'évaluer le risque individuel d'accident cardio-vasculaire et d'exploi- \\ ter toutes les autres possibilités de réduire le risque. Si le risque de maladie cardio- \\ vasculaire est inférieur à $10 \%$, la prescription de statines n'est pas indiquée. \\ L'organe responsable a pris connaissance du rapport et le soumet à une Post-Publi- \\ cation Review.
}

\section{Articles \\ interactifs

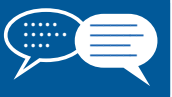 \\ Vous souhaitez commenter cet article? II vous suffit pour cela d'utiliser la fonc- tion «Ajouter un commen- taire» dans la version en ligne. Vous pouvez égale- ment consulter les remarques de vos confrères sous: www.bullmed.ch/ numero-actuel/ articles-interactifs/}

\section{Correspondance:}

Susanna Marti Calmell Secrétariat de l'organe responsable du

Swiss Medical Board Stampfenbachstrasse 30 CH-8090 Zurich Tél. 0432595211
Le terme de «statines» désigne les médicaments utilisés pour le traitement de l'hypercholestérolémie. Le cholestérol est un constituant important des membranes cellulaires; en conséquence, la présence de cholestérol est une condition préalable à une fonction physiologique normale. Toutefois, un taux de cholestérol trop élevé, conjugué à d'autres facteurs tels que, par exemple, le tabagisme et l'hypertension, représente un facteur de risque de maladies cardio-vasculaires. Le traitement par statines de patientes et patients souffrant déjà d'une maladie cardio-vasculaire (par ex. une maladie coronarienne) ou ayant subi un infarctus est largement admis au niveau international.

Dans son dernier rapport, le Conseil d'experts du Swiss Medical Board a examiné la question de savoir si, chez des personnes sans maladie cardio-vasculaire diagnostiquée («prévention primaire»), le nombre d'accidents cardio-vasculaires peut être diminué et la durée de survie et la qualité de vie améliorées grâce à l'administration de statines.

La littérature étudiée permet de conclure que les statines (c'est-à-dire la réduction du taux de cholestérol) peuvent diminuer le risque d'accidents cardio-vasculaires même chez les personnes sans trouble cardio-vasculaire diagnostiqué. Toutefois, un taux élevé de cholestérol ne représente qu'un facteur de risque parmi d'autres tels que, par exemple, le tabagisme, la surcharge pondérale ou l'hypertension. Il convient, dans ces cas, de réduire en premier lieu ces facteurs de risque, car l'administration de statines peut également avoir des effets indésirables; ceux-ci se manifestent la plupart du temps par des modifications des valeurs hépatiques et des dom- mages musculaires. Si l'on considère, en outre, le prix élevé des statines, le rapport coût-efficacité du traitement par statines en prévention primaire est défavorable pour les personnes présentant un risque faible d'accident cardio-vasculaire.

Le Conseil d'experts émet les recommandations suivantes:

- La prescription de statines en prévention primaire ne doit être prise en considération que lorsque les autres possibilités de réduire les facteurs de risque ont été épuisées.

- Le risque d'accident cardio-vasculaire doit être déterminé individuellement conformément aux directives en vigueur.

- La prescription de statines en prévention primaire n'est pas indiquée quand le risque d'accident cardio-vasculaire mortel est inférieur à 10\% (selon le score de l'ESC).

\footnotetext{
Post-Publication Review concernant le rapport «Statines en prévention primaire des maladies cardio-vasculaires»

Le rapport reflète l'appréciation du Conseil d'experts indépendant du Swiss Medical Board, basée sur l'évidence médicale actuelle. L'organe responsable du Swiss Medical Board a pris connaissance du rapport et souhaite inviter les milieux intéressés à une discussion dans le sens d'une Post-Publication Review. Les prises de position et les discussions peuvent être activées sur le site internet du Swiss Medical Board. Veuillez nous adresser votre avis à l'adresse suivante: info[at]medical-board.ch
} 